\title{
Ambulatory blood pressure and assessment of pacemaker function
}

\author{
R I JONES, * P M M CASHMAN, R S HORNUNG, H PRINCE, L BASSEIN, $\dagger$ \\ E B RAFTERY \\ From the Department of Cardiology, and the Divisions of Clinical Sciences, Bioengineering, and Computing \\ and Statistics, Northwick Park Hospital and Clinical Research Centre, Harrow, Middlesex
}

SUMMARY Ten patients in sinus rhythm with ventricular demand (VVI) pacemakers implanted for the sick sinus syndrome underwent 24 hour ambulatory blood pressure and electrocardiographic recording by a modified version of the Oxford system. Five patients had symptoms of dizziness or presyncope at the time of study and five were symptom free. The onset of pacing was associated with a fall in arterial blood pressure in both groups which was larger in the patients with symptoms, and in these patients the blood pressure recovery consequent on baroreflex activation was delayed by up to fifteen beats. In three of the patients with symptoms the original pacemaker was replaced by an atrioventricular pacing (DVI) device. This abolished symptoms and the initial fall and delayed recovery of blood pressure. Thus it appears that the development of symptoms of hypotension after the onset of ventricular pacing is determined by the rate of the baroreflex response. These symptoms and the haemodynamic consequences may be alleviated by dual chamber pacing.

A patient's electrocardiographic response to an implanted pacemaker is usually assessed in the outpatient clinic where the rate, pulse width, and other variables of the stimulus artefact are analysed at periodic intervals. Ambulatory electrocardiography has become an accepted additional method for the assessment of pacemaker function. ${ }^{12}$ Recent developments in Holter monitoring have facilitated recognition of the pacing stimulus artefact by detecting it separately and recording a marker pulse alongside the electrocardiogram on a two channel recorder. ${ }^{23}$ Such ambulatory recordings have proved valuable in various situations, such as detecting failure to capture, failure to sense, and myopotential inhibition.

Some patients, however, have symptoms despite pacemaker function which is electrocardiographically normal. It has been suggested that these can be alleviated in patients with carotid sinus

Requests for reprints to Dr E B Raftery, Cardiology Department, Northwick Park Hospital, Watford Road, Harrow, Middlesex.

Present addresses: *Cardiac Unit, Papworth Hospital, Cambridge; and +Cattedra e Servizio di Farmacologia Clinicia, Policlinico S Orsola, Via Massarenti 9, I-40138 Bologna, Italy.

Accepted for publication 11 February 1986 hypersensitivity by the implantation of an atrioventricular sequential (DVI) pacemaker. ${ }^{4}$ Alicandri et al investigated three patients with symptoms of near syncope after ventricular pacing, and demonstrated hypotension after the onset of pacing. ${ }^{5}$ Although these studies provided much useful information they were limited to the laboratory setting.

We have used the Oxford system for recording direct arterial pressure in ambulant subjects to study a group of patients in sinus rhythm paced for sick sinus syndrome with ventricular demand (VVI) pacemakers. Several patients had symptoms during our study; in three of these the original pacemaker was later replaced by a DVI device and a second study was performed.

We examined whether there was any systematic difference in the effects of pacing on blood pressure between those patients who complained of symptoms and those who did not. We particularly wished to observe the time course of any changes in blood pressure that might accompany the onset of pacing, and the compensatory response of the circulatory system. Ambulatory monitoring affords an ideal opportunity to study these effects quantitatively because many episodes containing the onset of pacing may be obtained from each patient. 


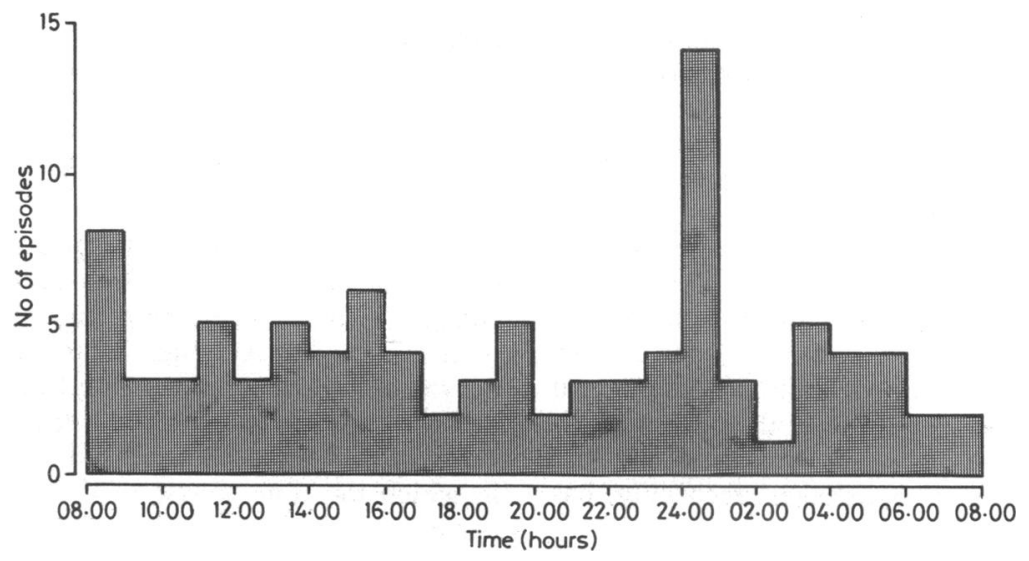

Fig. 1 Distribution of the selected episodes of the onset of pacing throughout 24 hours.

\section{Patients and methods}

Thirteen patients were recruited for this study and eighteen 24 hour recordings were performed. Data of adequate technical quality for analysis were obtained from ten patients in a total of thirteen 24 hour recordings. The patients gave their informed consent in writing and the study was approved by the hospital ethical committee. We studied only patients in whom sick sinus syndrome had been diagnosed before pacemaker implantation. None was taking any cardioactive drugs at the time of the study and none was in atrial fibrillation. In all of them a 24 hour ambulatory electrocardiographic recording was performed before the study to detect any evidence of electrical pacemaker malfunction.

Ambulatory recordings of direct arterial blood pressure and electrocardiogram were obtained with a modified Oxford.Medical Systems Medilog MKI
Recorder. The technique for ambulatory recording of direct arterial blood pressure has been described. ${ }^{6}$ After insertion of a brachial artery cannula, patients were allowed to be fully ambulant; they returned to the hospital every 12 hours for equipment checks and were asked to keep a diary of any symptoms that they experienced during the recording period. Four patients had symptoms of light headedness or presyncope during their first 24 hour recording. In three of these patients the original pacemaker was replaced by a DVI device and a second period of 24 hour recording was performed.

\section{RECORDING EQUIPMENT}

The standard Oxford Medilog electrocardiogram recorder does not register pacemaker signals accurately because of its limited high frequency capability. To distinguish reliably between paced and unpaced beats a pacing spike detector (Reynolds
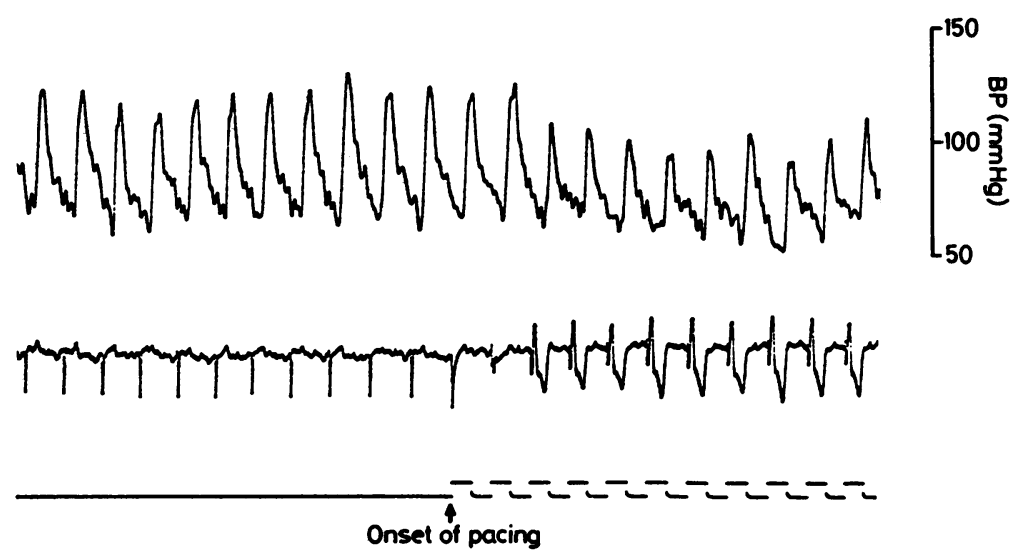

Fig. 2 Direct arterial blood pressure measurement, electrocardiogram, and pacing spike detector during the onset of VVI pacing in a patient without symptoms. 
Medical Electronics) was installed in the recorder. This module sensed the pacing activity in a surface electrocardiogram from an extra pair of electrodes that were positioned to maximise the spike amplitude while minimising other electrocardiogram components. The sensing electrode was usually placed over the V1 position and the reference electrode over the implanted pacemaker. The pacing spike detector module converted each spike into a pulse of $250 \mathrm{~ms}$ duration which was recorded on tape track 2 alongside the normal electrocardiogram on track $1 .^{3}$ An alternative version of this technique in which the same electrode pair was used for both electrocardiogram and pacemaker recording has been described elsewhere. ${ }^{7}$

\section{TAPE ANALYSIS}

The tapes were replayed at 25 times the recorded speed into a hybrid computing system ${ }^{8}$ that was slightly modified for pacemaker spike detection. The continuous blood pressure and pulse interval were extracted for each cardiac cycle. Track 2 of the tape was simultaneously scanned for paced beats. Each tape was first replayed in full to produce a trend chart of every paced beat in the 24 hours. From the trend chart an observer (HP) selected all episodes containing at least 20 beats of sinus rhythm followed by at least 20 beats of continuous pacing. At this stage she did not know which patients had symptoms. The episodes selected were then processed by the computer. A total of 100 episodes were analysed. Fig. 1 shows the distribution of these episodes throughout each 24 hours.

For each episode in which sinus rhythm changed to continuous pacing the beat by beat blood pressure and pulse interval values were printed out together with a marker indicating whether beats were paced or not. The signal timing was such that for each paced beat detected the corresponding printout showed the systolic pressure which occurred immediately after the pacing spike, the diastolic pressure which followed this systolic value, and the interval between this diastolic minimum and the preceding diastolic minimum. The same timing convention was used for unpaced beats. For these the $\mathbf{R}$ wave was used as the fiducial marker. For each episode, the printed results were checked by reference to a photographic chart writeout of the blood pressure, electrocardiogram, and pacing spike detector pulses (Fig. 2).

\section{STATISTICAL METHODS}

The 72 episodes initially selected during VVI pacing were divided into two groups: those from the patients with symptoms (40 episodes) and those from the symptom free patients (32 episodes). The length of the episodes varied from 40 to 80 cardiac cycles; beats were numbered negatively and positively about the onset of pacing (defined as beat zero).

The data were first analysed in terms of absolute values of blood pressure before, at the onset of pacing and 5,10 , and 15 beats after pacing. The individual blood pressure responses, and the mean changes in blood pressure during this period were then considered. Although this method of analysis provided some insight into the behaviour of blood pressure in response to pacing the wide individual variation in responses produced a smoothing effect in which the time course of the response was not apparent.

We therefore performed a further statistical analysis of each of the 72 episodes in which the beat by beat blood pressure was searched for significant rises or falls by regressing blood pressure on beat number within a window eleven points in length that could be moved along the entire length of the episode. At each position of the window the best fit was determined; a significant fall in blood pressure (blood pressure increase followed by decrease) was considered to be present if the regression yielded a negative quadratic component with a $t$ statistic greater than 2 (or equivalently an $\mathrm{F}$ statistic greater than 4). Similarly a significant rise in blood pressure (blood pressure decrease followed by increase) was said to have occurred if the regression yielded a significant positive quadratic component. If either type of response was found, the position of the midpoint of the window was noted. The window was then moved one beat to the right and the next regression was performed. By this means, the position of any significant peaks or troughs in the systolic data sequence could be determined relative to the onset of pacing, irrespective of the actual pressure values or heart rates. The results from all 72 episodes were then grouped for patients with and without symptoms. The rises and falls in blood pressure were plotted separately and for each of these four subgroups the observed and expected numbers of significant responses were compared by a $\chi^{2}$ test. When we calculated the expected distribution of responses we took into account the differing lengths of the raw data episodes. Therefore, for each position relative to the onset of pacing, the expected number of positive responses was evaluated as the total positive responses at all positions multiplied by the proportion of episodes containing valid data at the current position. The expected distribution of negative responses was similarly calculated.

We also compared ( $\chi^{2}$ test) the number of significant rises and falls in blood pressure in the patients with symptoms and those without. In three 
Ambulatory blood pressure and assessment of pacemaker function

Table Clinical data in 10 patients

\begin{tabular}{|c|c|c|c|c|c|}
\hline Patient No & Age & Sex & Pacemaker type & Time since implant & Symptoms \\
\hline $\begin{array}{l}1^{\star} \\
2(a)^{\star} \\
3^{(b)} \\
4 \\
5(a)^{\star} \\
(b) \\
7^{7} \\
8(a)^{\star} \\
9^{(b)} \\
10^{\star}\end{array}$ & $\begin{array}{l}69 \\
71 \\
70 \\
72 \\
55 \\
68\end{array}$ & $\begin{array}{l}\mathbf{M} \\
\mathbf{F} \\
\mathbf{M} \\
\mathbf{M} \\
\mathbf{M} \\
\mathbf{M} \\
\mathbf{M} \\
\mathbf{M}\end{array}$ & $\begin{array}{l}\text { CPI Microlith } \\
\text { CPI Microlith } \\
\text { DVI Medtronic } \\
\text { CPI Microlith } \\
\text { Precilith } \\
\text { CPI Microlith } \\
\text { DVI Cyberlith } \\
\text { CPI Microlith } \\
\text { CPI Microlith } \\
\text { CPI Microlith } \\
\text { DVI Microlith } \\
\text { Unilith } \\
\text { CPI Microlith }\end{array}$ & $\begin{array}{l}2 \mathrm{yr} \\
3 \mathrm{yr} \\
2 \mathrm{mnth} \\
2 \mathrm{mnth} \\
3 \mathrm{mnth} \\
1 \mathrm{yr} \\
2 \mathrm{mnth} \\
3 \mathrm{yr} \\
4 \mathrm{mnth} \\
6 \mathrm{mnth} \\
2 \mathrm{mnth} \\
5 \mathrm{yr} \\
6 \mathrm{mnth}\end{array}$ & $\begin{array}{l}\text { Presyncope } \\
\text { Presyncope } \\
\text { Nil } \\
\text { Nil } \\
\text { Nil } \\
\text { Presyncope } \\
\text { Nil } \\
\text { Nil } \\
\text { Nil } \\
\text { Presyncope } \\
\text { Nil } \\
\text { Nil } \\
\text { Presyncope }\end{array}$ \\
\hline
\end{tabular}

Patients undergoing re-study ( $a$ and $b$ ).

$\star$ Patients with symptoms.
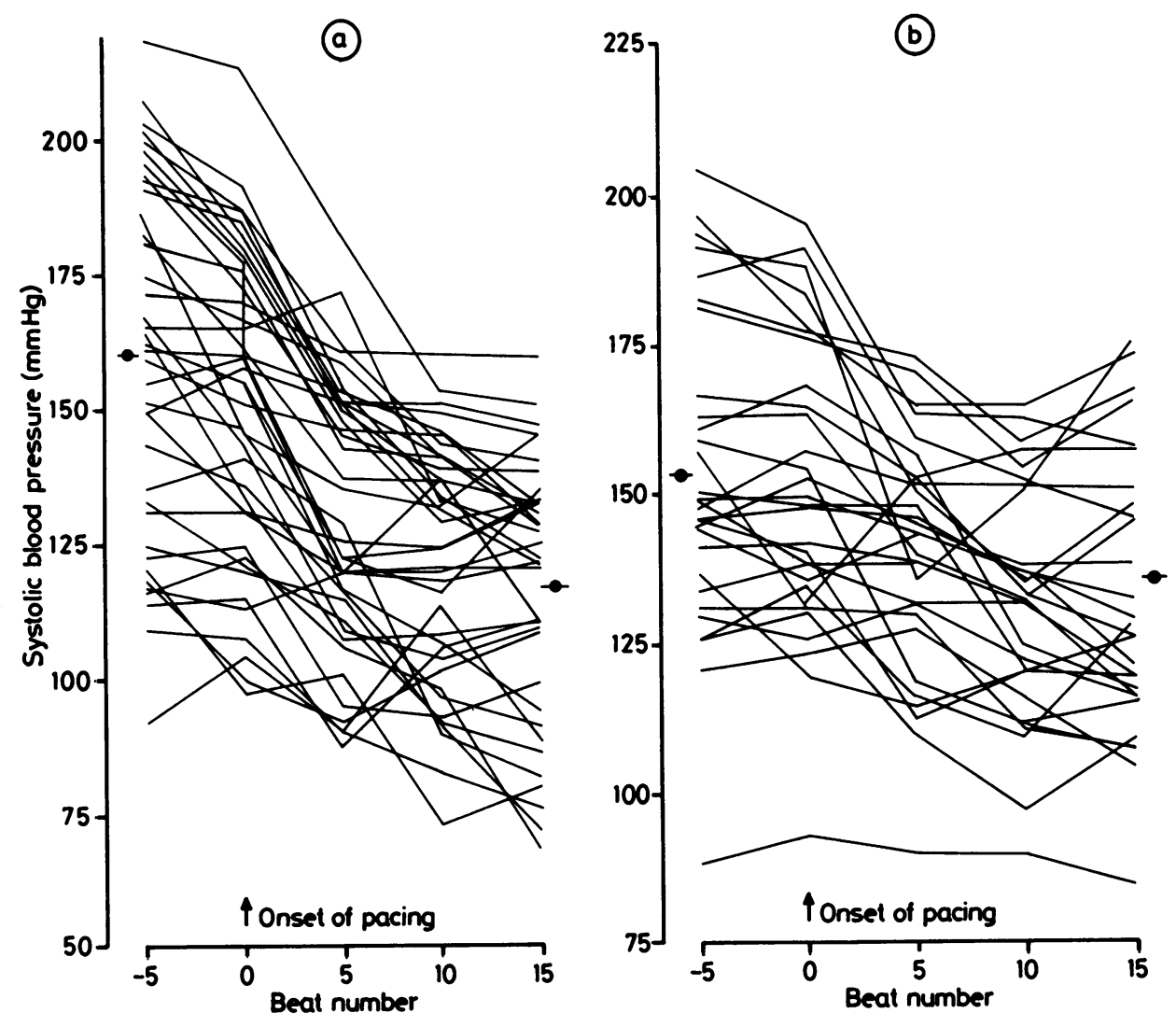

Fig. 3 Individual changes in blood pressure during each of the selected episodes in patients with symptoms (a) and in those without (b). 

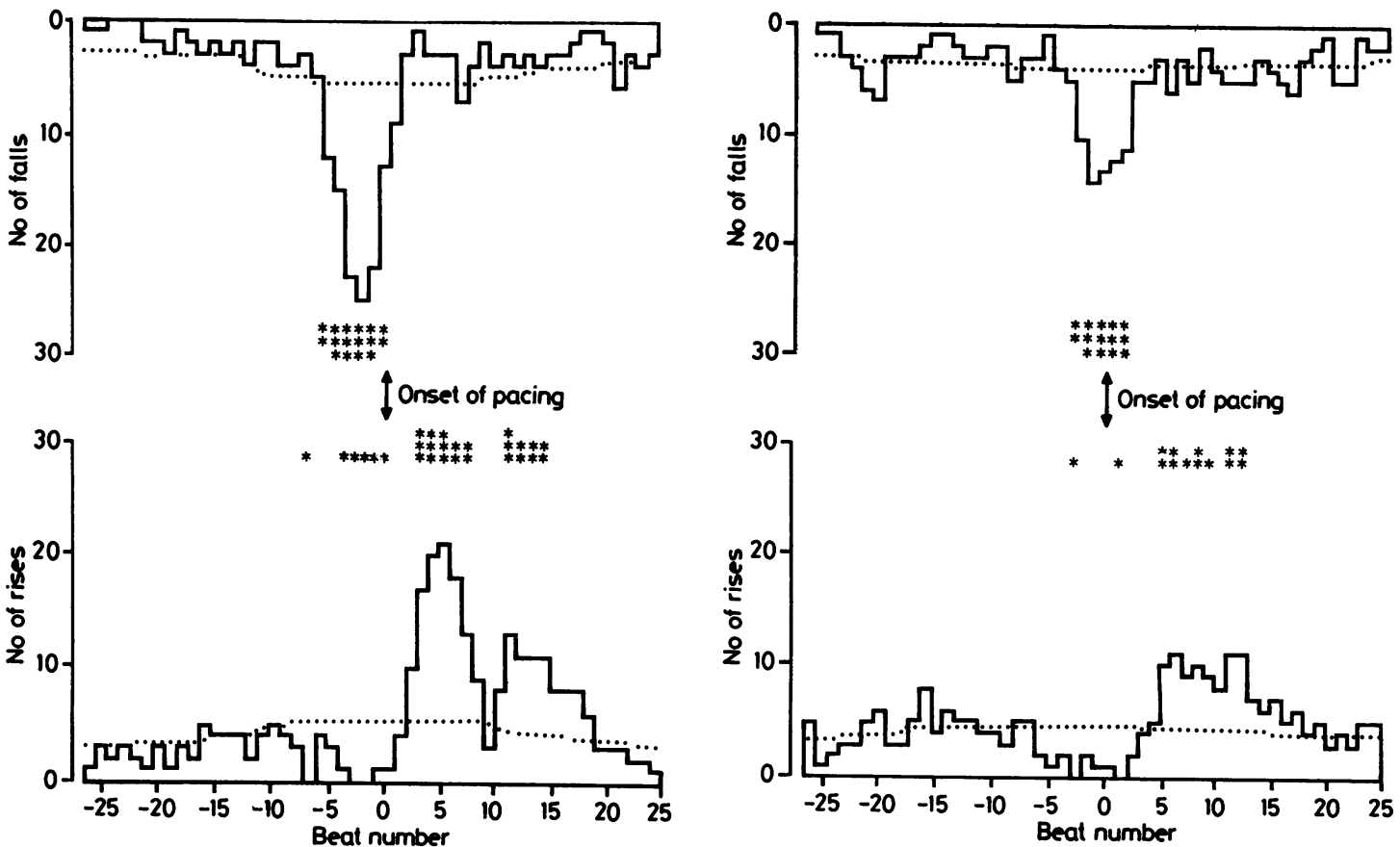

Fig. 4 Effects of VVI pacing on systolic blood pressure in five patients with symptoms. The number of episodes expected (see text) is indicated by the broken line. ${ }^{\star} p<0.05$;

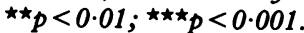

Fig. 5 Effects of VVI pacing on systolic blood pressure in five symptom free patients. The number of episodes expected is indicated by the broken line. ${ }^{\star} p<0.05 ;{ }^{\star \star} p<0.01$; $\star \star \star p<0.001$

patients results before (27 episodes) and after (28 episodes) replacement of their VVI pacemaker with a DVI device were also compared.

\section{Results}

Five recordings were excluded from the analysis, two because of pump artefact, one because of only very short episodes of sinus rhythm, and two because the pacemaker detector failed to trigger. Figure 1 shows the distribution of the selected episodes throughout the 24 hours; there was an increase at 24.00 hours, presumably associated with retiring to bed.

The Table shows the clinical data for the ten patients. Of the two patients who had symptoms at the time of the first study and who were not restudied, one had episodes of hypoglycaemia which were thought to be responsible for his symptoms and one declined a change of pacemaker. The individual changes in blood pressure during each of the selected episodes were plotted for patients with symptoms and for those without (Fig. 3). The mean fall in blood pressure was $40(21) \mathrm{mm} \mathrm{Hg}$ in those with symptoms and $18(15) \mathrm{mm} \mathrm{Hg}$ in those without

symptoms $(p<0.001)$. Figure 4 shows the effect of pacing on systolic blood pressure in patients with symptoms, and Fig. 5 results in patients without symptoms. Pacing induced a fall in blood pressure in both groups. This was followed by a compensatory rise after five beats. Patients with symptoms, however, had a considerable number of delayed compensatory responses, unlike those without symptoms. A direct statistical comparison showed that there were significantly fewer rises and falls in blood pressure in the symptom free patients and significantly more in those with symptoms $(p<0.001)$.

Figure 6 shows the response of systolic blood pressure during VVI pacing in three of the patients with symptoms. The pattern of response (a fall followed by two different rates of recovery) was maintained in this group. After the original pacemaker was replaced by a DVI device both the fall in blood pressure at the onset of pacing and the subsequent compensatory rise were abolished (Fig. 6), as were the patients' symptoms. In both groups, with and without symptoms, a similar, although less pronounced, response was seen in diastolic blood pressure. 
(a)

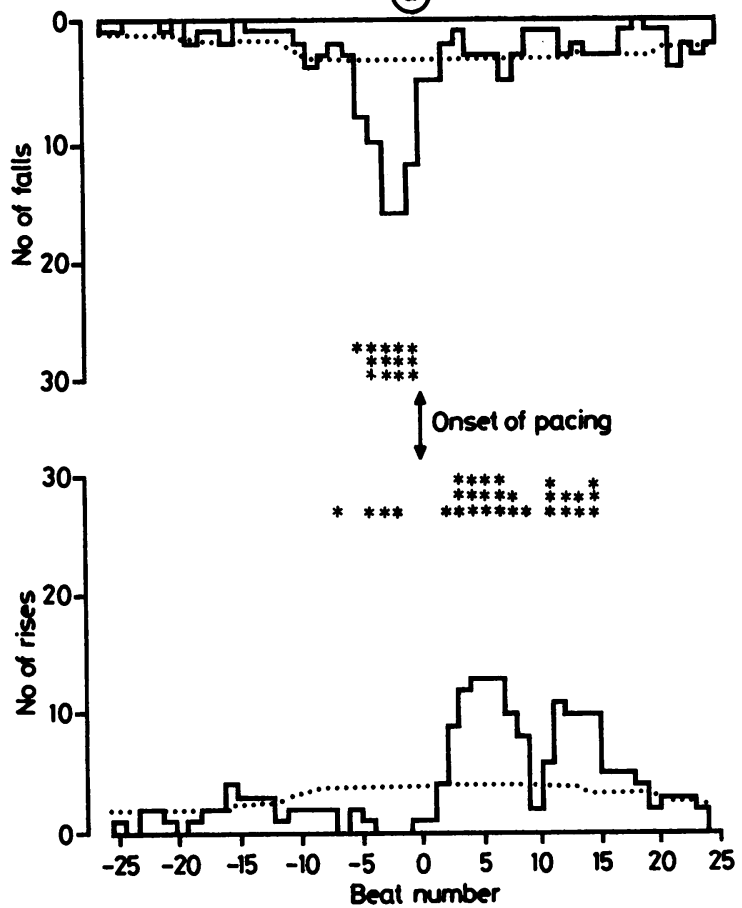

(b)
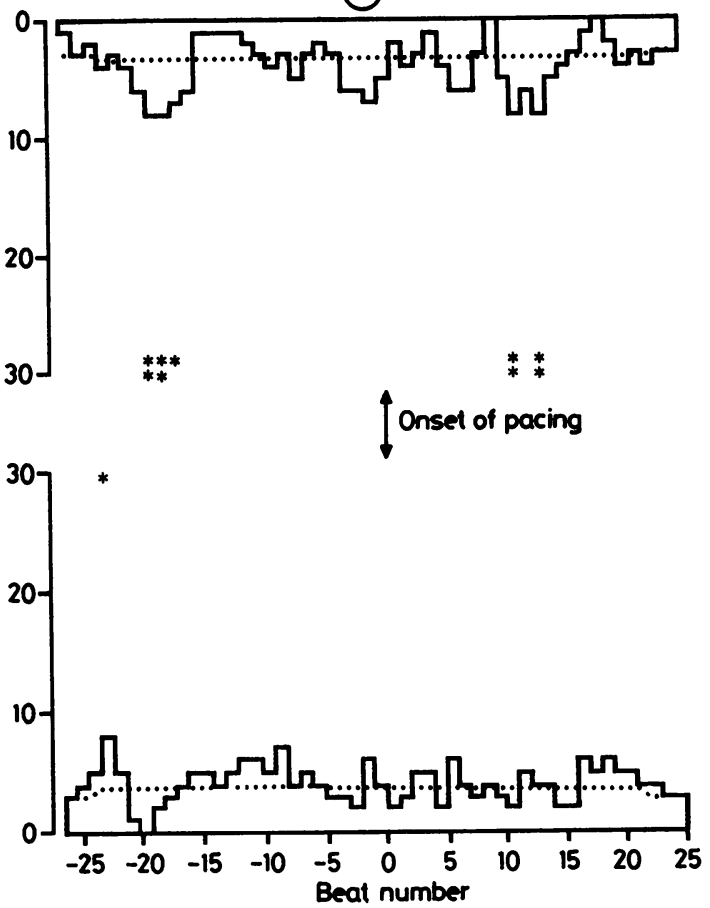

Fig. 6 (a) Effects of VVI pacing on systolic blood pressure in three patients with symptoms. (b) Effect in same patients after substitution of a DVI pacemaker. The number of episodes expected is indicated by the broken line. ${ }^{\star} p<0.05$; ${ }^{\star} p<0.01$; $\star \star \star p<0.001$.

\section{Discussion}

The pacemaker syndrome or pacemaker effect, with the development of hypotension after the onset of ventricular pacing, is well recognised; however, its incidence and importance is disputed. Cohen et al recently suggested that atrial transport was of clinical importance in $19(7.3 \%)$ of 260 consecutive patients undergoing implantation of a permanent pacemaker. ${ }^{9}$ Their patients developed symptoms ranging from dizziness and syncope to congestive heart failure and shock. Alicandri et al have suggested that the level of arterial blood pressure during VVI pacing is determined by two opposing reflexes, the baroreflex which attempts to compensate for a reduction in cardiac output, and an atrial distension reflex that leads to a reduction in peripheral vascular resistance. $^{5}$

Using the technique of ambulatory monitoring and selecting only patients with underlying sinus rhythm, we obtained a large number of episodes of a change from sinus to paced rhythm for further study. Analysis of the time course of the blood pressure response confirmed that the onset of ventricular pacing produced a fall in blood pressure in all patients. In patients with symptoms, however, two types of recovery pattern were seen with recovery delayed by fifteen beats ( \pm 13 seconds) during some episodes. These findings suggest that as well as the fall in blood pressure it is the duration of hypotension induced by an impaired baroreflex response which is important in the development of symptoms.

Two of the three patients who complained of symptoms experienced dizzy spells during their first 24 hour recording. The patients' diaries were not considered sufficiently accurate to relate symptoms to specific haemodynamic events. Separate analysis of the blood pressure responses during VVI pacing in the three patients with symptoms in whom the original pacemaker was later replaced showed an identical pattern of recovery. These findings imply that both types of recovery (immediate or delayed) may occur in an individual patient, depending on the circumstances at the time of onset of pacing. The benefit of implanting an atrial electrode and DVI generator in these three patients was clearly demonstrated. Their symptoms were alleviated, and analysis of the blood pressure response during pacing showed that the initial fall in blood pressure, 
and hence the need for baroreflex activation, was abolished.

It is possible that the time course of the recovery in blood pressure is in part related to the rate at which atrioventricular desynchronisation occurs, and the duration of hypotension may be influenced by the rate at which atrioventricular synchrony is restored. The heart rates before the onset of pacing were similar in the two patient groups. Unfortunately the quality of electrocardiographic recording in most patients was inadequate for further analysis of atrioventricular synchronisation. Although it would be difficult to perform invasive haemodynamic studies in all patients complaining of presyncope after pacemaker implantations, the results of this study suggest that an impaired baroreflex response to a fall in cardiac output is of importance in determining the development of symptoms. This group of patients seems to benefit from the substitution of a dual chamber pacing device.

We thank Professor C E Rossiter for advice and guidance on the statistical analysis procedures, and Dr M C Petch for his helpful comments during the preparation of this paper.

\section{References}

1 Ward DE, Camm J, Spurrell RAJ. Ambulatory monitoring of the electrocardiogram in the pacemaker sur- veillance clinic in patients with presyncope. In: Stott FD, Raftery EB, Sleight P, Goulding L, eds. ISAM. London: Academic Press, 1977: 33-5.

2 Famularo MA, Kennedy HL. Ambulatory electrocardiography in the assessment of pacemaker function. Am Heart f 1982; 104: 1086-96.

3 Murray A, Jordan RS, Gold RG. Pacemaker assessment in the ambulant patient. $B r$ Heart $\mathcal{F} 1981$; 46: 531-8.

4 Morley CA, Perrins EJ, Grant P, Chan SL, McBrien DJ, Sutton R. Carotid sinus syncope treated by pacing: analysis of persistent symptoms and the role of atrioventricular sequential pacing. Br Heart $\mathcal{f} 1982$; 47: 411-8.

5 Alicandri C, Fouad FM, Tarazi RC, Castle C, Morant $V$. Three cases of hypotension and syncope with ventricular pacing: possible role of atrial reflexes. $A m \mathcal{F}$ Cardiol 1978; 42: 137-42.

6 Millar Craig MW, Hawes D, Whittington J. A new system for recording ambulatory blood pressure in man. Med Biol Eng Comput 1978; 16: 727-31.

7 Walsh T, Goldberg AD, Mehta S. A method for continuous monitoring of pacemaker function. In: Stott FD, Raftery EB, Goulding L, eds. ISAM. London: Academic Press, 1979: 493-6.

8 Cashman PMM, Stott FD, Millar Craig MW. Hybrid system for fast data reduction of long term blood pressure recordings. Med Biol Eng Comput 1979; 17: 629-35.

9 Cohen SI, Frank HA. Preservation of active atrial transport: an important clinical consideration in cardiac pacing. Chest 1982; 81: 51-4. 